\title{
Preface for the special issue on recent advances and future perspectives of new drug development and therapeutics on cardiovascular diseases
}

\author{
You Mie Lee ${ }^{1}$
}

Published online: 15 March 2019

(c) The Pharmaceutical Society of Korea 2019

Cardiovascular diseases (CVDs) are the number one cause of death globally with about 17.9 million deaths in 2016, accounting for $31 \%$ of the global mortality rate, $85 \%$ of which was due to heart attack and stroke. Early detection of CVDs, high blood pressure, diabetes, and hyperlipidemia is important to prevent from further risk and should be treated with appropriate counseling and pharmacotherapy. Cancer can be categorized as another form of vascular disease having hypervascularization and vascular abnormalities. Over the past few decades, many promising results have been generated using innovative technological approaches in the field of CVDs, ranging from biology to new drug development, which are beautifully reviewed in this special issue. As recent research outcomes in novel mechanisms and technologies have unraveled new horizons for the development of therapeutic drugs for CVDs, in this special issue, the combination of imaging technology with drug development, cell therapy using stem cells, mechanism of CVDs and development of therapeutic drugs by noncoding RNAs are highly encouraged to be reviewed. This information provides new insights into the understanding and control of signaling pathways involved in communication between bioactive small molecules/biomolecules and their partner molecules to express biological phenotypes related to CVDs. Moreover, this will unravel a new gateway to drug discovery by providing both new or existing drugs and target proteins relevant for treating other diseases of

You Mie Lee

lym@knu.ac.kr

1 Lab of Vascular Homeostasis Regulation, Molecular Pathophysiology, College of Pharmacy, Kyungpook National University, 80 Daehak-ro, Buk-gu, Daegu 41566, Republic of Korea interest. Moreover, this special issue will reveal recent research targets for CVDs and broaden the possibilities and horizons for new therapeutic targets. In this sense, this special issue is an invaluable forum of new drug and target discovery for CVDs.

This special issue of Archives of Pharmacal Research (APR) on "Recent advances and future perspectives of new drug development and therapeutics on cardiovascular diseases" emphasizes the prominent role of biological mechanisms for drug discovery as a platform for novel biologically relevant drug discovery targets as well as small molecules. As such, it consists of seven noteworthy reviews covering new trends of almost all research area of drug discovery on CVDs. As the first part, three papers dealt with the ischemic cardiovascular diseases. At the beginning, "Cell signaling and biological pathway in cardiovascular diseases" reviewed by Cheong-Whan Chae and Yoo-Wook Kwon highlighted coronary artery disease and its related signalling molecules, including adipokines as crucial mechanistic target. "Current pharmacotherapies for atherosclerotic cardiovascular diseases" reviewed by Jong-Gil Park and Goo Taeg Oh summarized atherosclerotic cardiovascular disease (ASCVD) in which limitation and possibility of lipid-modifying strategies and further treatments for inflammatory microenvironment as a promising target are outlined. Jae Ho Kim and his colleagues reviewed "Role of stem cell mobilization in the treatment of ischemic diseases" in which recent application and interests of stem cells for therapeutic tools in ischemic diseases that induce mobilization of stem cells from the bone marrow is highlighted.

As the second part, three papers figured out the vascular diseases ranging from cancer angiogenesis to vascular complications of the endocrinological disorders, such as calcification and diabetes. Sung Min Cho and 
Ho Jeong Kwon highlighted the roles of sphingolipids in cancer angiogenesis and reviewed the strategies to utilize and target the sphingolipid metabolism, especially by targeting acid ceramidase, which can modulate cancer apoptosis and angiogenesis, prominent value of dual aspects to inhibit tumor growth. Young-Kook Kim and Kook Hyun summarized the recent researches on "Diverse roles of noncoding RNAs in vascular calcification" in which the potential technologies to modulate the noncoding RNAs, including microRNAs for therapeutic purpose especially for the vascular calcification are highlighted. In-Kyu Lee and his colleagues revealed recent literature for biochemical/cellular targets being utilized for the treatment of diabetic microvascular diseases, such as polyol and diacylglycerol-protein kinase C pathways, oxidative stress, non-enzymatic glycation and the formation of advanced glycation end products, and immune dysregulation/ inflammation.

Finally, one paper reviewed by Jin-Hui Yoon and Yong Jeong addressed about recent technology of vascular imaging especially in neurovascular system which is the most complicated and not much adventured. However, imaging for neurovascular diseases can be a representative tool for various vascular disorders and provide the solution to link between cognitive disorders and vascular homeostasis. Recent in vivo imaging technology is rapidly developing owing to the development of optical technology as well as software programs to calculate images. This review precisely reviewed the recent advances of in vivo imaging and future directions for promoting neurovascular disease research.

It was my great privilege and pleasure to work with all distinguished scientists, experts and authors who contributed their precious and invaluable knowledge and experience to this special issue with the reviews. On the behalf of all contributors, I would like to express my special appreciation to Dr. Seok-Yong Lee, the editors-inchief of APR. Last but not the least, I sincerely believe that the special volume of this journal will provide a better understanding of the methodology and development of new drug discovery for the treatment of cardiovascular diseases to the scientists in this field as well as other relevant fields.

\section{Publisher's Note}

Springer Nature remains neutral with regard to jurisdictional claims in published maps and institutional affiliations. 